\title{
Editorial
}

\section{Multifault Detection, Diagnosis, and Prognosis for Rotating Machinery}

\author{
Zhixiong Li $\mathbb{D}^{1}$ and Grzegorz M. Królczyk $\mathbb{D D}^{2}$ \\ ${ }^{1}$ University of Wollongong, Wollongong, NSW, Australia \\ ${ }^{2}$ Politechnika Opolska, Opole, Poland \\ Correspondence should be addressed to Zhixiong Li; zhixiong.li@ieee.org
}

Received 21 March 2018; Accepted 21 March 2018; Published 1 August 2018

Copyright (C) 2018 Zhixiong Li and Grzegorz M. Królczyk. This is an open access article distributed under the Creative Commons Attribution License, which permits unrestricted use, distribution, and reproduction in any medium, provided the original work is properly cited.

As professionals working in the field of condition monitoring and fault diagnosis, we know that reliable recognition of fault type and assessment of fault severity are essential for decision making in condition-based maintenance of rotating machinery. In engineering practice, the mechanical systems of rotating machinery are often subject to concurrent faults on the same component or different components, which make the examination of both the fault types and severities more challenging. Popular intelligent algorithms such as artificial neural networks (ANNs) are proven effective in identifying different fault patterns while "physical meanings" of the identification process are often missed due to blackbox of intelligent algorithms. Alternatives such as multimodal decomposition approaches enable decoupling the hybrid faults into submodes. Each submode describes a single fault in the hybrid faults. As a result, the "physical meanings" of the identification process can be revealed using the multimodal decomposition approaches. This special issue looks at latest multimodal decomposition approaches for multifault detection, diagnosis, and prognosis on rotating machinery.

The article by K. Chen et al. (Wuhan University of Technology, China) is a good place to begin this special issue as the authors introduced the variational mode decomposition (VMD) as the multimodal decomposition approach to detect multiple faults in rotor systems. The decomposed vibration signals using VMD can be used to extract effective features for multifault detection. The authors evaluated the performance of the proposed method using experimental data.

In another article, G. An and H. Li from Mechanical Engineering College in China developed a multimodal decomposition approach based on fundamental component extraction (FCE) algorithm for multifault detection of rotor systems. The failures in stator and rotor can be effectively identified by the proposed FCE method.

In another two articles, H. Li et al. (State Key Laboratory of Mechanical Transmission, China) presented an image tensor extraction method for rotor fault diagnosis and $\mathrm{K}$. Chen et al. (Wuhan University of Technology, China) introduced an integrated approach of ensemble empirical mode decomposition and deep briefs network to diagnose gear multiple faults. The authors conducted experimental testing to evaluate the performance of the proposed approaches.

$\mathrm{Y}$. Li et al. is a good place to conclude this special issue as the authors proposed a new method based on variational mode decomposition and Gath-Geva clustering time series segmentation to extract the degradative feature of rolling element bearings and predict the bearing failures. The effectiveness of the proposed bearing degradation prediction method was verified by two case studies.

\section{Acknowledgments}

I would like to thank the authors for submitting their work to this special issue. I would also like to express my gratitude to the reviewers and the guest editors for their help and support with producing this issue. 


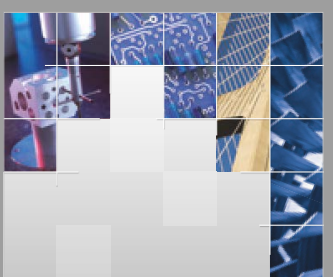

\section{Enfincering}
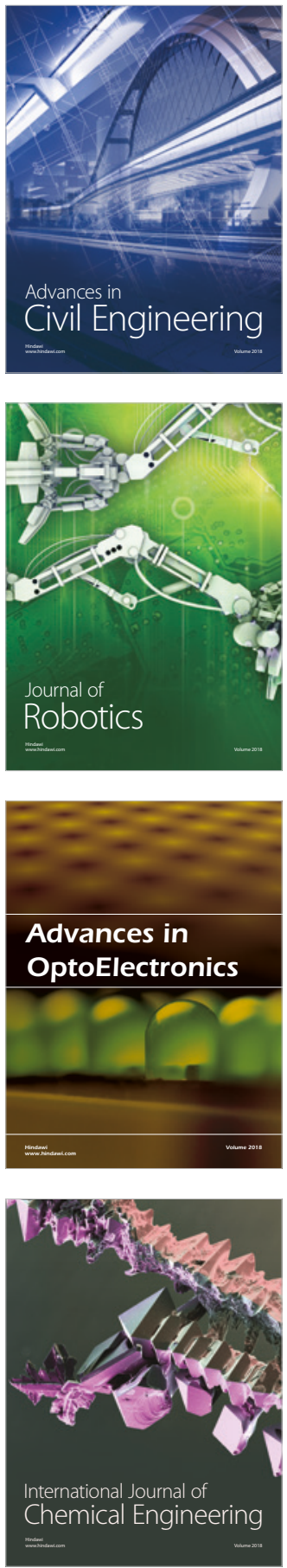

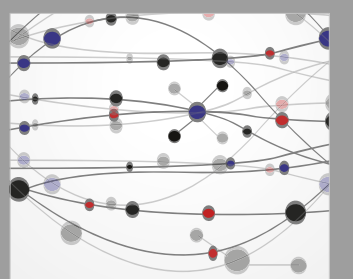

\section{Rotating \\ Machinery}

The Scientific World Journal

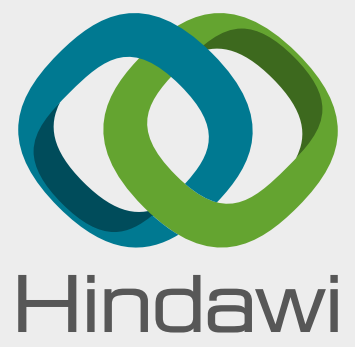

Submit your manuscripts at

www.hindawi.com
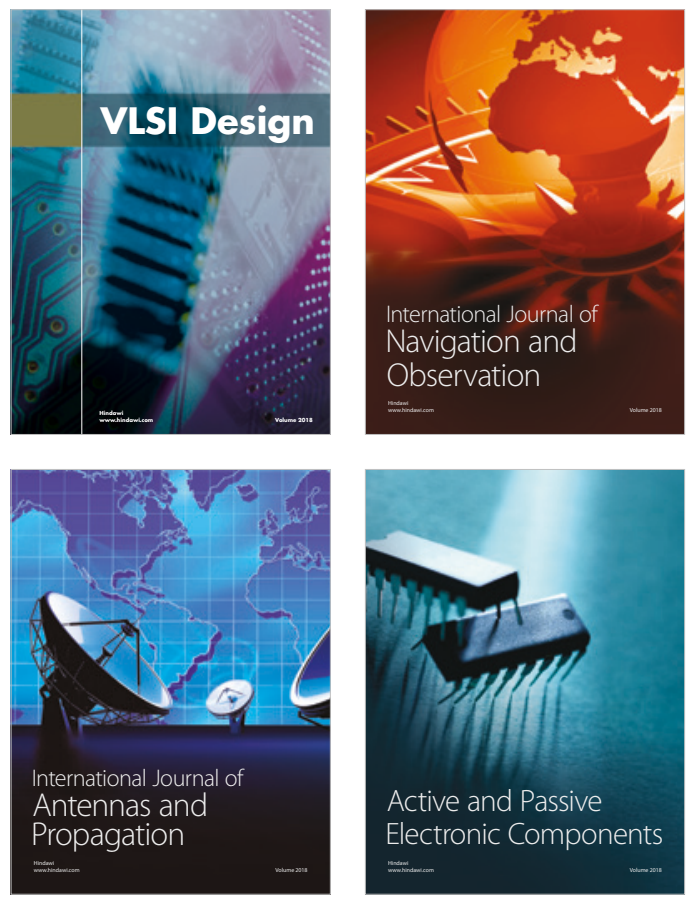
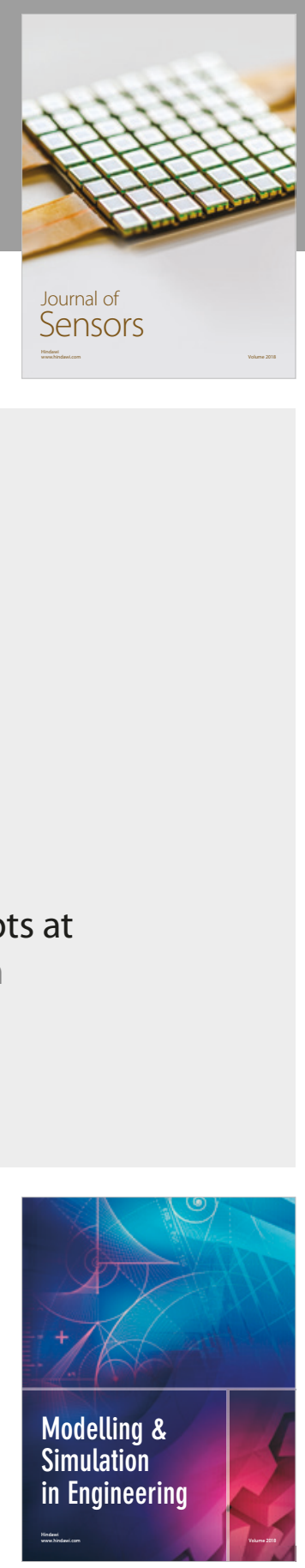

\section{Advances \\ Multimedia}
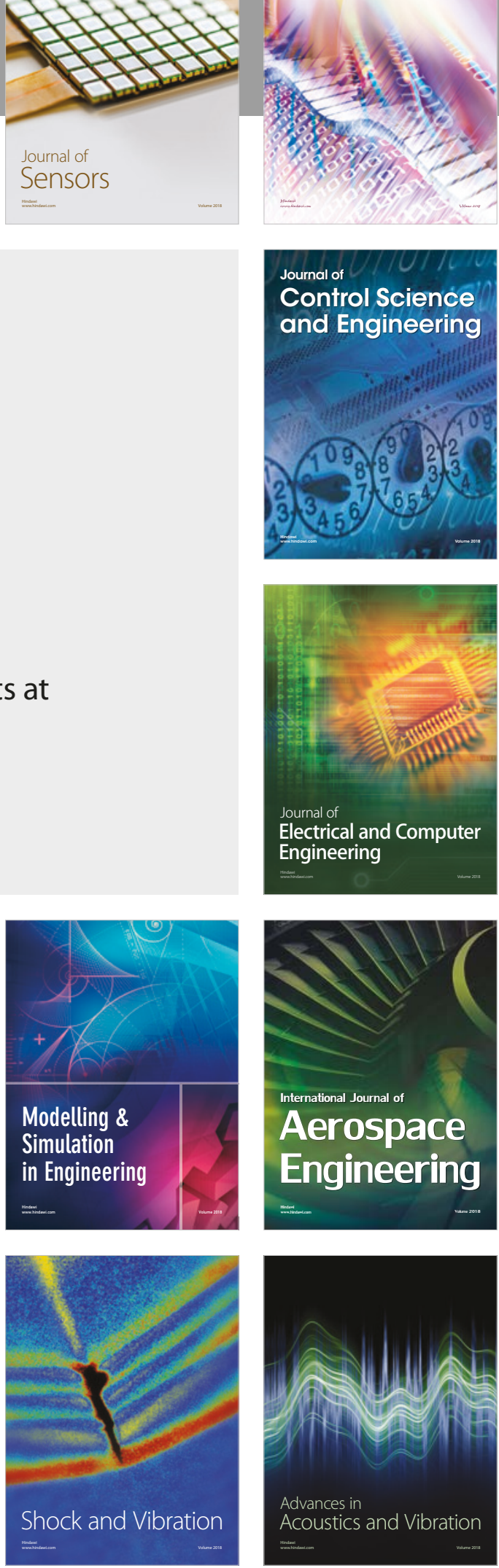